\title{
Cancer Stem Cell Signaling during Repopulation in Head and Neck Cancer
}

\author{
George D. Wilson, ${ }^{1,2}$ Bryan J. Thibodeau, ${ }^{2}$ Laura E. Fortier, ${ }^{2}$ \\ Barbara L. Pruetz, ${ }^{2}$ Sandra Galoforo, ${ }^{1}$ Brian Marples, ${ }^{1}$ Andrew M. Baschnagel, ${ }^{3}$ \\ Jan Akervall, ${ }^{2,4}$ and Jiayi Huang ${ }^{5}$ \\ ${ }^{1}$ Department of Radiation Oncology, William Beaumont Hospital, Royal Oak, MI 48703, USA \\ ${ }^{2}$ Beaumont BioBank, William Beaumont Hospital, Royal Oak, MI 48703, USA \\ ${ }^{3}$ Department of Human Oncology, University of Wisconsin Carbone Cancer Center, Madison, WI 53792, USA \\ ${ }^{4}$ Department of Otolaryngology, William Beaumont Hospital, Royal Oak, MI 48703, USA \\ ${ }^{5}$ Department of Radiation Oncology, Washington University School of Medicine in St. Louis, St. Louis, MO 63110, USA
}

Correspondence should be addressed to George D. Wilson; george.wilson@beaumont.edu

Received 8 July 2015; Revised 7 October 2015; Accepted 15 November 2015

Academic Editor: Ahmad Waseem

Copyright (C) 2016 George D. Wilson et al. This is an open access article distributed under the Creative Commons Attribution License, which permits unrestricted use, distribution, and reproduction in any medium, provided the original work is properly cited.

\begin{abstract}
The aim of the study was to investigate cancer stem signaling during the repopulation response of a head and neck squamous cell cancer (HNSCC) xenograft after radiation treatment. Xenografts were generated from low passage HNSCC cells and were treated with either sham radiation or $15 \mathrm{~Gy}$ in one fraction. At different time points, days 0,3 , and 10 for controls and days $4,7,12$, and 21, after irradiation, 3 tumors per group were harvested for global gene expression, pathway analysis, and immunohistochemical evaluation. 316 genes were identified that were associated with a series of stem cell-related genes and were differentially expressed ( $p \leq 0.01$ and 1.5-fold) at a minimum of one time point in UT-SCC-14 xenografts after radiation. The largest network of genes that showed significant changes after irradiation was associated with CD44, NOTCH1, and MET. c-MET and ALDH1A3 staining correlated with the changes in gene expression. A clear pattern emerged that was consistent with the growth inhibition data in that genes associated with stem cell pathways were most active at day 7 and day 12 after irradiation. The MET/CD44 axis seemed to be an important component of the repopulation response.
\end{abstract}

\section{Introduction}

Head and neck squamous cell cancers (HNSCCs) are a heterogeneous group of malignancies that originate in the mucosal lining of the upper aerodigestive tract. Despite advances in therapy, survival rates have remained static for many years [1].

The heterogeneity of HNSCC, as evidenced by histological, phenotypical, and karyotypical analyses $[2,3]$, has been mainly ascribed to the process of clonal expansion [4]. However, there is an increasing awareness that not all heterogeneity among cancer cells is the result of genetic variability and that, within a single tumor clone, cells have significantly different abilities to proliferate and form new tumors. This has led to the hypothesis that most cells in a cancer have a limited ability to divide and only a small subset of phenotypically distinct cells, the cancer stem cells (CSCs), have the capacity to self-renew and form new tumors [5]. The presence of cells with "stem-like" properties has been observed in HNSCC using a variety of different approaches [6-11].

For advanced inoperable HNSCC, treated with radiotherapy or chemoradiation, locoregional progression is the principal cause of treatment failure and cancer-related death. If tumor repopulation after therapy is a property of CSCs then the response of this population to radiation will be a critical constraint for curability. Several studies have provided evidence that CSCs are more resistant to radiation than their non-CSCs counterparts in a variety of cancers [12-15] and an association with chemoresistance has been reported in many studies including HNSCC [16]. 
In this study, we developed a model of local failure and repopulation in a HNSCC xenograft using a subcurative dose of radiation and studied the changes in protein expression of known stem cell-related genes as well as stem cell-related signaling pathways using global gene expression at key time points during the tumor response.

\section{Materials and Methods}

2.1. Cell Line, Xenografts, and Irradiation. The UT-SCC-14 cell line was obtained from Dr. R. Grénman, University of Turku, Finland, and was selected from a large panel of cell lines derived from primary and recurrent tumors from the head and neck region. The cell line has been maintained at low passage number such that it maintains phenotypic and morphological characteristics similar to the primary tumor which was a T3N1M0, moderately differentiated, HPV negative squamous cell carcinoma of the oral tongue. The experimental protocol was approved by the William Beaumont Hospital Animal Care Committee. Four- to 6week-old female nude NIH III mice were used in these studies. Mice were caged in sterile housing in groups of five and were fed a diet of animal chow and water ad libitum. Xenografts were established by harvesting UT-SCC-14 cells in mid-log phase growth and injecting them subcutaneously into the flank of the mice, at a concentration of $2 \times 10^{6}$ cells per $100 \mu \mathrm{L}$ of Matrigel (BD, Franklin Lakes, NJ). Tumor volume was measured by digital calipers and calculated using the standard formula $(\pi a b 2) / 6$, where $a$ is the largest and $b$ is the smallest diameter. When the tumor volume reached a value of $300-400 \mathrm{~mm}^{3}$, animals were randomly assigned to the experiment groups. Tumors were measured three times each week. The endpoint of the experiment was when the tumors grew to a volume of $3,000 \mathrm{~mm}^{3}$. Animals were irradiated with a Faxitron Cabinet X-Ray System, Model 43855F (Faxitron $\mathrm{X}$-Ray, Wheeling, IL), at a dose rate of $0.69 \mathrm{~Gy} / \mathrm{min}$, tube voltage of $160 \mathrm{KVp}$, and current of $4 \mathrm{~mA}$. Animals were immobilized (without anesthetic) in a custom-designed jig that only exposed the hind flank to the radiation beam.

2.2. Experimental Design. Nine xenografts were randomized to receive sham treatment (control group) and twelve were randomized to receive $15 \mathrm{~Gy}$ (RT group). Groups of 3 mice from each treatment cohort were sacrificed at different time points after treatment. The control time points were selected during exponential growth of the unirradiated tumor and were not linked to the irradiated time points which were based on the regrowth characteristics of the tumors to yield observations during growth inhibition, transition to regrowth. At each time point the tumor was rapidly excised, bisected, and half snap-frozen and stored at $-80^{\circ} \mathrm{C}$ whilst the other half was fixed in zinc formalin. For the control group, tumors were harvested at days 0,3 , and 10 after reaching the starting volume of $400-500 \mathrm{~mm}^{3}$. For the RT group, tumors were harvested at days $4,7,12$, and 21 after treatment.

2.3. Isolation of RNA and Gene Expression. Laser capture microdissection was used to isolate cells from the peripheral regions of the tumor based on our previous observation of central necrosis after radiation treatment [17]. Frozen tissue samples were embedded in OCT (Tissue-Tek; Sakura Finetek, USA) and $8 \mu \mathrm{m}$ sections were cut and mounted onto PEN (polyethylene naphthalate) membrane glass slides (two sections per slide). Regions of periphery were identified on corresponding $\mathrm{H} \& \mathrm{E}$ slides of the tissue sections. The stained slides were microdissected within 2 hours of sectioning using an Arcturus ${ }^{\text {XT }}$ Microdissection System (Molecular Devices) onto CapSure Macro LCM Caps (Molecular Devices).

RNA isolation was carried out using RNeasy Plus Micro Kit (Qiagen, Valencia, CA). RNA concentration was determined on a ND-8000 spectrophotometer (NanoDrop Technologies, Inc., Wilmington, DE) and quality assessed on a Model 2100 Bioanalyzer (Agilent Technologies, Santa Clara, CA). High-quality RNA (i.e., RIN > 9.5) was used for the experiments. Differential mRNA expression analysis between cell populations was performed according to the GeneChip Whole Transcript (WT) Sense Target Labeling Assay Protocol (Affymetrix Inc., Santa Clara, CA) using 100 ng of RNA from each specimen. The fragments were hybridized overnight with the Human Exon 1.0 ST Array and then scanned with a GeneChip Scanner 3000. The GEO accession number is GSE61573.

2.4. Data Analysis. The .CEL files containing the raw intensity data from the Affymetrix GeneChip arrays were imported into Partek Genomics Suite (version 6.6 beta, build 6.11.1115) and normalized using the robust multichip average with a guanine-cytosine content background correction, quantile normalization, $\log _{2}$-transformation, and median polish probeset summarization. Exons were then summarized to genes using the average of the probesets. Differentially expressed genes were identified using 1-way ANOVA comparing the samples from a given irradiated time point to the controls.

Gene set enrichment analysis (GSEA) and pathway analyses were performed using Pathway Studio 10.3 (Elsevier, New York, NY, USA). GSEA identifies highly regulated categories by considering all genes without any prefiltering based upon $p$ value or fold change [18]. The expression microarray data was also analyzed using Pathway Studio's Sub-Network Enrichment Analysis (SNEA) tool [19, 20]. Pathway Studio utilizes MedScan, the literature mining program that searches publicly available literature such as PubMed for relationships between entities [21]. A subnetwork consists of a single seed (i.e., disease or cell process) and genes associated with this seed by regulation of/by the seed [22]. The expression microarray dataset is interrogated with no prior significance filtering, and enrichment of the subnetwork is determined by both the level of regulation in the network and the size of the network. The visualized subnetwork was limited to include only those genes that met $p \leq 0.01$ and 1.5 -fold change.

2.5. Immunohistochemistry and Image Analysis. $4 \mu \mathrm{m}$ sections were cut, deparaffinized, and hydrated by passing through xylene followed by graded alcohol to distilled water. Antigen retrieval was achieved using antigen unmasking solution ( $\mathrm{pH}$ 9.0) (Vector Labs, Burlingame, CA). 
Endogenous peroxide was blocked with Envision Flex peroxidase blocking solution (Dako, Carpinteria, CA) and the nonspecific staining blocked with CAS universal blocking solution (Life Technologies, Grand Island, NJ). Rabbit polyclonal antibodies for CD44 and ALDH1A3 were obtained from Abcam and used at a dilution of 1:50; c-MET clone 8F11 (Vector Laboratories Inc., Burlingame, California, USA) was diluted $1: 100$ and incubated at $42^{\circ} \mathrm{C}$ for 2 hours; (Abcam, Cambridge, MA) CD44 and ALDH1A3 were diluted 1:50 and $1: 100$, respectively, in antibody diluent containing PBS ( $\mathrm{pH} 7.4$ ), BSA, and $0.05 \%$ sodium azide and incubated for 60 minutes. After washing, the sections were incubated for 20 minutes using a dual rabbit/mouse polymer link detection kit (Dako) and visualized using diaminobenzidine and counterstained with hematoxylin. The sections were then dehydrated with a gradient of ethanol to xylene and coverslipped.

Images were captured at $10 \mathrm{x}$ magnification on a Nikon 90i microscope (Nikon, Melville, NY) equipped with a Nikon DS-Fil digital camera and Nikon Elements software. Ten images were captured from the peripheral areas of each tumor sample. The percent area of the image that stained positively for each protein was analyzed using ImageJ software (NIH). Images were split using the RGB function, an autothreshold was set on the blue image, and the area showing positive DAB staining was calculated using the measure function.

2.6. Statistical Analysis. Statistical analysis was carried out using Student's $t$-test. Data are presented as mean \pm SE. A probability level of a $p$ value of $<0.05$ was considered significant.

\section{Results}

3.1. Tumor Characteristics, Growth Rate, and Response to Radiation. Figure 1 shows the time course of the radiation response in the UT-SCC-14 xenografts. The untreated UTSCC-14 xenografts had a volume doubling time of $4.8 \pm$ 0.7 days whilst the irradiated tumors showed a period of profound growth arrest until day 12 after which they transitioned into repopulation. In a different aspect of this present study, we showed that early radiation necrosis (days 4-12) was characterized by central coagulative necrosis with pyknotic nuclei whilst late radiation necrosis (day 12 onwards) was characterized additionally by extensive necrosis with fragmentation and dystrophic calcifications [17]. The histological studies demonstrated that repopulation of the tumor occurred from the peripheral region, and this was the reason why immunohistochemical and gene expression analysis was restricted to only this region in the control and treated animals by the use of laser capture microdissection.

\subsection{Immunohistochemical Staining of Stem Cell-Associated} Proteins. Figure 2(a) shows the immunohistochemical staining pattern and changes after irradiation of 3 of the genes with known association to stem cells in HNSCC; in Figure 2(b), the quantitative analysis of these genes is presented. CD44 was mainly associated with the cell surface and tended to stain cells in the outer layers of the tumor islands formed

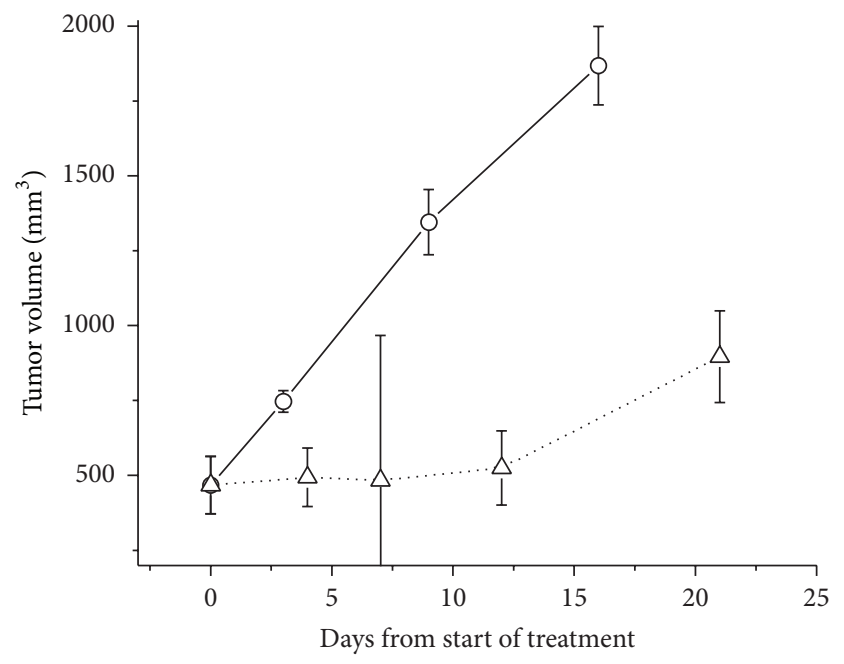

FIGURE 1: The tumor growth response of UT-SCC-14 xenografts to a dose of $15 \mathrm{~Gy}$. Flank tumors were either irradiated with $15 \mathrm{~Gy}(\triangle)$ or sham-irradiated $(\mathrm{O})$ and their growth was monitored over time with calipers.

by the UT-SCC-14 cells; no staining was observed in the stroma. Following radiation, there was not a great change in the intensity of staining but the number of positive cells fell steadily at day $4(p=0.0201)$ and day $7(p=$ $0.0123)$ and to a nadir at day $12(p=0.0014)$ after which expression levels recovered. The staining pattern became more predominantly associated with the outermost cell layers of the tumor islands. In control tumors there was a reduction in CD44 expression as tumor volume increased. Interestingly, CD44 expression decreased in control tumors as a function of time. Histologically, this was associated with a tendency for the larger tumors to show differentiation and increased keratinization.

ALDH1A3 staining was cytoplasmic and showed a diffuse pattern throughout the tumors where complete tumor islands were often positive with little evidence of any preferential peripheral staining within those islands. After irradiation ALDH1A3 increased to a maximum on day $7(p=0.047)$ and remained elevated for the period of observation. In some areas cells became more intensely stained with ALDH1A3 after irradiation.

Intrinsic levels of c-MET were relatively low in UTSCC-14 xenografts and showed a staining pattern which was similar to ALD1A3 being diffuse and cytoplasmic. The levels of c-MET increased at day $7(p=0.0357)$ and reached a maximum at day $12(p=0.005)$ after which they declined rapidly to control levels.

3.3. Global Gene Expression Changes Associated with Stem Cell-Related Signaling. In Table 1, Pathway Studio software was used to identify genes associated with a series of stem cell-related genes CD44, ALDH, MET, NOTCH1, BMI1, OCT3/4, NANOG, SOX2, and CD133, which were differentially expressed ( $p \leq 0.01$ and 1.5 -fold) at a minimum of one time point in UT-SCC-14 xenografts after radiation. 

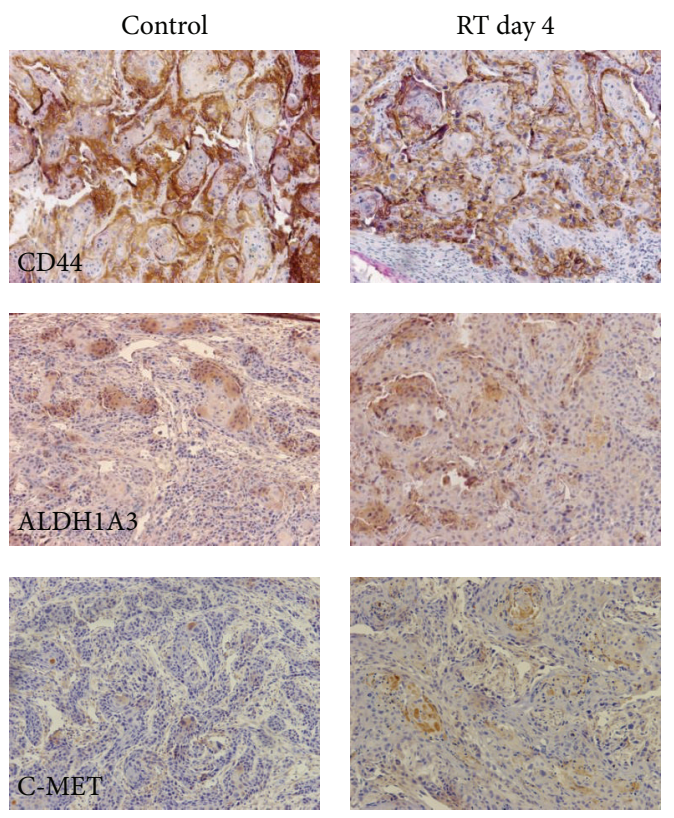

RT day 7
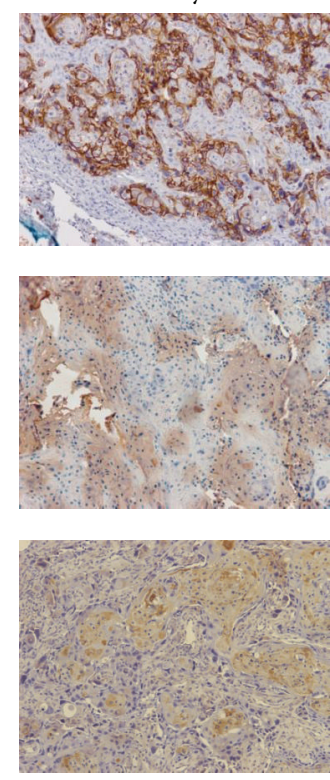

(a)
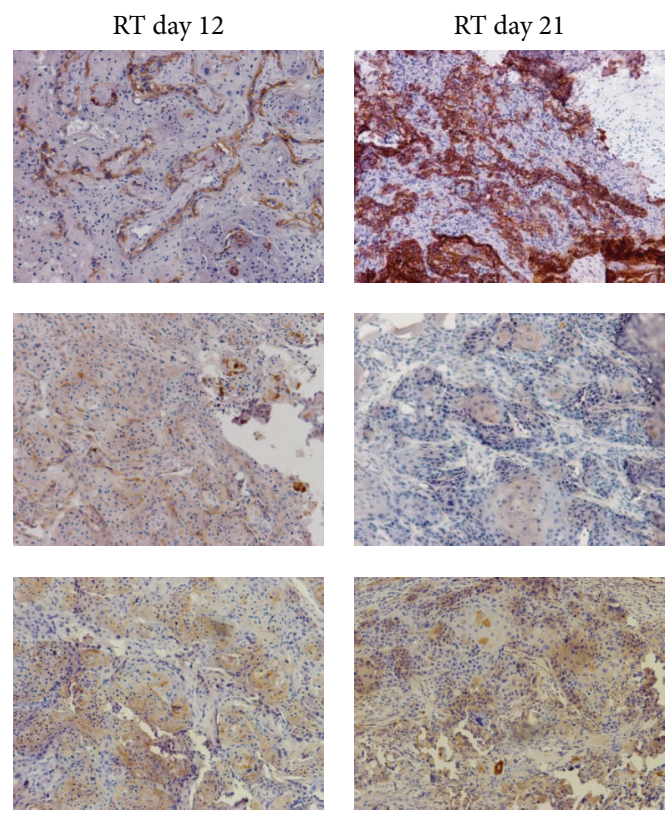
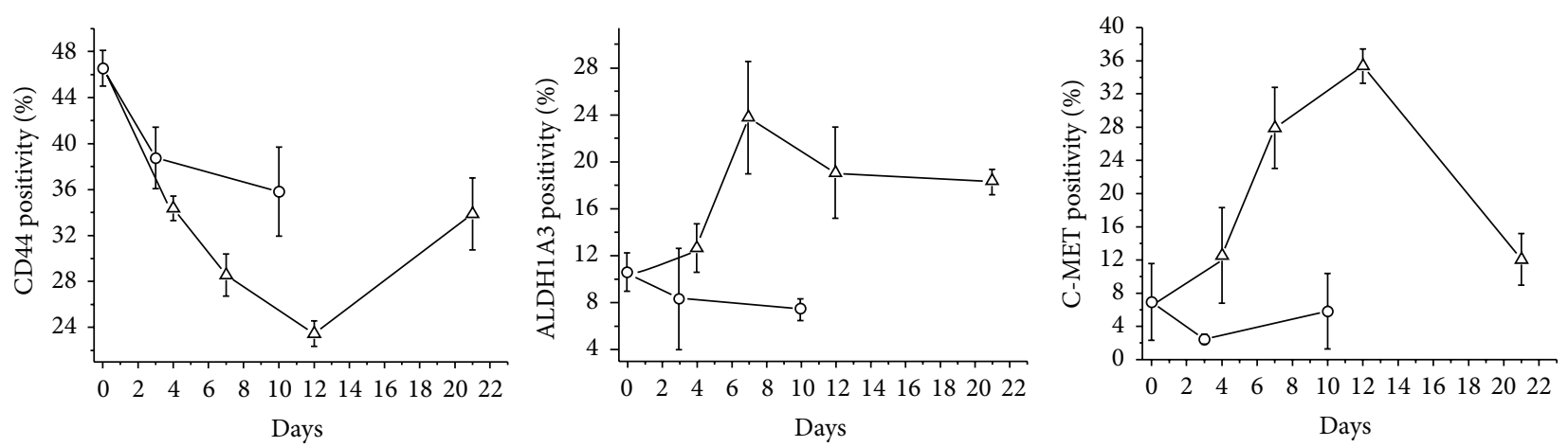

(b)

FIGURE 2: Immunohistochemical staining for CD44, ALDH1A3, and c-MET (a) and image analysis quantification of protein expression (b) after radiation (RT) treatment. CD44 was mainly associated with the cell surface and tended to stain cells in the outer layers of the tumor islands; its expression decreased after radiation but recovered between days 12 and 21. ALDH1A3 staining was cytoplasmic and showed a diffuse pattern throughout the tumors. After irradiation ALDH1A3 increased to a maximum on day 7 and remained elevated for the period of observation. In some areas cells became more intensely stained with ALDH1A3 after irradiation. c-MET showed a staining pattern which was similar to ALDH1A3 being diffuse and cytoplasmic. The levels of c-MET increased at day 4 and reached a maximum at day 12 after which they declined rapidly to control levels $(\times 20)$.

The associated genes could be of any category including ligand, transcription factor, positive or negative regulator, or receptor. Pathway Studio identified 316 genes that met these criteria for the 9 stem cell-related genes, many of which were shared between the target genes. The largest network of genes that showed significant changes after irradiation was associated with CD44, NOTCH1, and MET. The smallest network of genes was associated with $A L D H$. There was a clear pattern that emerged in which genes associated with stem cell pathways were most active at day 7 and day 12 after irradiation. At day 7, the tumors are still in a state of profound growth inhibition whilst at day 12 the transition into regrowth is becoming apparent (Figure 1). At days 4, 7, and 12, there was a higher proportion of genes that were upregulated in the stem cell-associated pathways but, interestingly, at day 21 (during active regrowth) more downregulated genes were represented.

\subsection{Specific Gene Expression Changes Associated with Stem} Cell Signaling. Three genes, CTNNB1, MMP9, and NOTCH1, were identified in the pathways of all of the nine stem cellassociated genes. However, CTNNB1 was only upregulated at day 4; MMP9 was upregulated at days 12 and 21, whilst NOTCH1 was downregulated at days 12 and 21. Three genes, IL6, IL8, and SMAD2, were represented in 8 of the 9 signaling pathways. SMAD2 was only upregulated at day 7 whilst both IL6 and IL8 were upregulated at day 12. Seven genes were present in 7 of the 9 pathways and included ICAM1, LIF, 
TABLE 1: Genes differentially expressed ( $p \leq 0.01$ and 1.5-fold) at a minimum of one time point in UT-SCC-14 xenografts after radiation.

\begin{tabular}{lccccccccc}
\hline \multirow{2}{*}{ Gene } & $n$ & \multicolumn{2}{c}{ Day 4} & \multicolumn{3}{c}{ Day 7} & \multicolumn{2}{c}{ Day 12} & \multicolumn{2}{c}{ Day 21 } \\
& & $\uparrow$ & $\downarrow$ & $\uparrow$ & $\downarrow$ & $\uparrow$ & $\downarrow$ & $\uparrow$ & $\uparrow$ \\
\hline CD44 & 62 & 5 & 3 & 20 & 12 & 28 & 11 & 3 & 4 \\
ALDH & 9 & 2 & 0 & 2 & 4 & 3 & 1 & 1 & 1 \\
MET & 48 & 7 & 1 & 16 & 8 & 25 & 9 & 4 & 5 \\
OCT3/4 & 37 & 6 & 1 & 10 & 5 & 18 & 4 & 4 & 4 \\
BMI1 & 16 & 1 & 0 & 2 & 7 & 6 & 1 & 0 & 1 \\
NOTCH1 & 66 & 7 & 4 & 19 & 14 & 29 & 11 & 1 & 4 \\
NANOG & 29 & 5 & 2 & 7 & 4 & 15 & 4 & 4 & 3 \\
SOX2 & 29 & 3 & 1 & 8 & 5 & 12 & 3 & 1 & 2 \\
CD133 & 20 & 2 & 1 & 3 & 4 & 9 & 3 & 0 & 3 \\
Total & $\mathbf{3 1 6}$ & $\mathbf{3 8}$ & $\mathbf{1 3}$ & $\mathbf{8 7}$ & $\mathbf{6 3}$ & $\mathbf{1 4 5}$ & $\mathbf{4 7}$ & $\mathbf{1 8}$ & $\mathbf{2 7}$ \\
\hline
\end{tabular}

Using Partek Genomics software and Pathway Studio, genes that were differentially expressed at a minimum of one time point after radiation with a $p$ value $\leq 0.01$ and fold change (negative or positive) of 1.5 or greater were identified for each of the CSC-associated genes. For each gene, $n$ represents the total number of identified genes in each pathway whilst the $\uparrow$ and $\downarrow$ arrows for each day after radiation represent the number of genes which were upregulated or downregulated, respectively.

MAPK8, MET, SPP1, TGFA, and TGFBR2. MET and TGFA were upregulated at key days 7 and 12. LIF1 and SPP1 were upregulated at day 12 whilst MAPK8 was upregulated at day 7 only.

3.5. Specific Gene Expression Changes Associated with CD44. Supplemental Table 1 in Supplementary Material available online at http://dx.doi.org/10.1155/2016/1894782 shows genes significantly altered at one time point or more known to be associated with CD44 signaling. It is clear that the greatest activity centers on days 7 and 12 . On day 7 the genes showing the greatest fold change were as follows: TIMP3 (+2.44), TIMP2 (+2.39), IGHMBP2 (+2.36), MMP3, KHDRB53, TGFA, and WIPF1 all showed between 1.90and 1.99-fold increases whilst PTGES, PLAUR, CTSB, MET, and VANGL1 all showed greater than 1.75-fold increases. The top downregulated genes were IGFBP5 (-3.40), PTTG1 $(-3.07)$, EGR1 (-2.55), and FASN (-2.54). On day 12 more genes showed significant alterations and higher levels of fold change. IL8 (+6.00), HAS2 (+5.35), ICAM1 (+3.51), PLAUR (+3.32), LIF $(+3.13)$, and LYN $(+3.07)$ were the most significantly upregulated genes but TIMP2, MMP9, HBEGF, CXCL16, SLC7A11, MME, EPCAM, CTSS, PLAU, and TGFBR2 all showed greater than 2-fold upregulation. Of the downregulated genes IGFBP5, BCAM, and NOTCH1 showed the greatest downregulation. Interestingly HRAS was downregulated on days 7,12 , and 21 .

3.6. Specific Gene Expression Changes Associated with MET. Supplemental Table 2 shows that MET shares many common genes with CD44 and shows a similar pattern of the most active gene expression changes taking place on days 7 and 12. Some of the key genes upregulated on day 7 were WNT7A (+2.46), TIMP3 (+2.44), TIMP2 (+2.39), MMP3
$(+1.99)$, TGFA $(+1.91)$, and PLAUR $(+1.84)$. MET itself was upregulated 1.76 -fold and was also upregulated at day 12 1.86 -fold. Several genes were highly upregulated in the cMET pathway at day 12 including $L C N 2(+10.0), I L 8(+6.0)$, ICAM1 (+3.51), PLAUR (+3.32), LIF (+3.13), LYN (+3.07), TIMP2 (+2.92), MMP9 (+2.82), HBEGF (+2.76), and WNT7A $(+2.25)$. Some of the most noticeable downregulated genes were HRAS, FASN, EGR1, FGFR3, and NOTCH1.

3.7. Specific Gene Expression Changes Associated with NOTCH1. Supplemental Table 3 shows that NOTCH1 also shares many common genes with CD44 and MET and reflects the changes in WNT7A, TIMP3, TIMP2, MMP3, TGFA, PLAUR, and MET at day 7. Other genes upregulated in this pathway at day 7 included FHL1 $(+2.45)$, NFATC2 $(+2.04)$, and ENO2 (+2.03). Similarly, at day 12 many mutual genes were upregulated including IL8, ICAM1, PLAUR, LIF, TIMP2, MMP9, HBEGF, and WNT7A; other notable upregulated genes included IL1RL1, FHL1, IL6ST, TRIB3, PMAIP1, RIPK2, PLAU, TGFBR2, TGFA, BIRC3, and BMI1. IGFBP5, NOTCH1, JAG2, HRAS, and STAT6 were notable genes downregulated at day 12 .

3.8. Specific Gene Expression Changes Associated with Other Stem Cell-Related Genes including ALDH, BMI1, CD133, NANOG, POU5F1, and SOX2. The ALDH, BMI1, CD133, NANOG, POU5F1, and SOX2 pathways had fewer members which were significantly altered after radiation (Supplemental Tables 4-9). ALDH1A3 was the key gene in the ALDH pathway that was upregulated at day $7(+2.18)$, day $12(+4.18)$, and day $21(+2.75)$. The BMI1 pathway showed nothing remarkable outside of genes altered in many of the other pathways. There was a similar conclusion with the $C D 133$ pathway. The NANOG and POU5F1 (OCT 3/4) pathways shared some similar upregulated genes which were not associated with the other major signaling pathways. These included TOR1AIP2 which was upregulated at day $4(+3.5)$, day $7(+4.27)$, and day $12(+3.75)$ and PMP22 which was upregulated at day 7 $(+3.32)$, day $12(+3.02)$, and day $21(+1.95)$. SOX2 signaling was relatively unremarkable with most genes being represented in the other major pathways.

3.9. Common Cell Processes Associated with Stem Cell Signaling after Radiation Treatment. Figure 3 shows cell processes that were linked with all nine stem cell-associated genes. Apoptosis and differentiation were almost universally negatively regulated by the genes whilst cell growth, cell proliferation, cell migration, and epithelial-to-mesenchymal transition were positively regulated by the stem cell-associated genes.

3.10. Epithelial-to-Mesenchymal Transition. Epithelial-tomesenchymal transition (EMT) appears as a common cell process in Figure 3, and it functions to diversify cell types during embryogenesis and also allows epithelial cells to acquire a migratory, mesenchymal-like phenotype during wound healing. Inducing EMT in HNSCC cells correlates with the emergence of CSCs and vice versa. In Supplemental Table 10, we have listed the genes showing $p \leq 0.01$ and at 


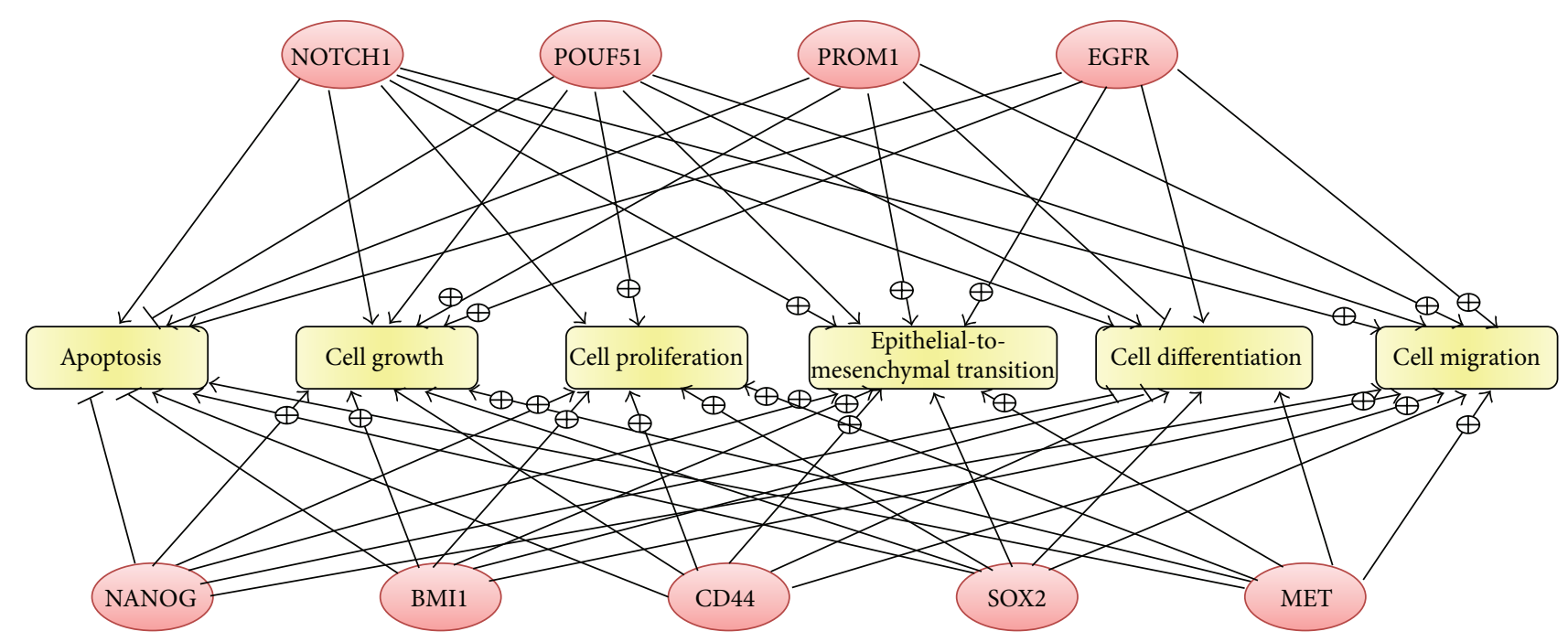

Figure 3: Cellular processes common to all nine cancer stem cell- (CSC-) associated genes. Pathway Studio was used to identify known relationships and cellular processes that are common between the CSC-associated genes. The $\bigoplus$ symbol on the arrow represents positive regulation of the process whilst a - through the arrow represents negative regulation.

least a 1.5 -fold change after irradiation. There is a similar pattern to the stem cell-associated genes where significant changes increase from day 4 through day 7 , peak at day 12 , and decline by day 21. Many of the genes are shared among the stem cell-associated gene pathways. In Figure 4, we have linked the pathways of the stem cell-associated genes with EMT presenting common genes found in at least 5 of the 9 CSC markers and EMT with a differential expression of $p \leq 0.01$ and at least a 1.5 -fold change. CTNNB1 and MMP9 were the only genes linked to all the subnetworks whilst $I L 8$, IL6, and SMAD2 were found in 9 of the ten subnetworks and ICAM1, SPP1, TGFA, LIF, TGFBR2, and MAPK3 were found in 8 subnetworks.

\section{Discussion}

It is generally accepted that CSC populations with selfrenewal and differentiation capacities exist within HNSCC $[16,23]$. A better understanding of the mechanisms that govern the dynamics of HNSCC CSCs is needed to unravel their importance and role in treatment resistance and disease progression. In this study, we developed a model which interrogated the molecular changes associated with known stem cell linked genes during the repopulation/regrowth of a HNSCC xenograft after a subcurative radiation treatment. The underlying rationale for this approach was that if the CSCs were important in treatment resistance, they would dominate the repopulation response as a single dose of $15 \mathrm{~Gy}$ would kill approximately $90 \%$ of the cells in the tumor.

Many of the genes including CD44 [24], BMI1 [25], cMET [26], NOTCH1 [27], ALDH1 [28], and SOX2 [29] have been associated with poor prognosis in HNSCC. However, the genes were specifically chosen in this study because they have all been associated with CSCs in HNSCC and other cancers. CD44 is a multifunctional transmembrane glycoprotein that is a receptor hyaluronic acid but can also interact with several additional molecules such as fibronectin, fibrinogen, laminin, galectin-8, collagen, chondroitin sulfate, and osteopontin. It has been proposed that HA binding to CD44 promotes its association with EGFR as well as EGFR phosphorylation. CD44 is the most commonly used cellsurface marker for CSCs across multiple tumor types and has been consistently acknowledged as CSC marker in HNSCC $[30,31]$. Prince and colleagues were the first to show $\mathrm{CD} 44^{+}$ cells were highly tumorigenic and successfully propagated in serial transplantation studies [10]. CD44 expression has been associated with poor prognosis in HNSCC [24]. The aldehyde dehydrogenase family of enzymes participates in retinoic acid biosynthesis and is an integral component of squamous epithelia differentiation. Cells which express high levels of ALDH1, extracted from primary HNSCC, were shown to be more tumorigenic in establishing xenografts than cells with low expression [6] and other studies have shown that ALDH1 overexpression correlates with poor prognosis [32]. The combination of $\mathrm{CD}_{4} 4^{+}$and $\mathrm{ALDH}^{\text {high }}$ expression further identified primary HNSCC cells that showed enhanced xenotransplantation efficiency [33]. cMET is a receptor tyrosine kinase that has been implicated in the progression of HNSCC [34] and associated with worse response to treatment and prognosis [26]. It has also been implicated in CSCs as a subset of c-MET ${ }^{+}$cells were shown to have enhanced tumorigenicity which was more pronounced when cells which were positive for both c-MET and CD44 were transplanted [35]. NOTCH1 signaling ordinarily drives keratinocyte differentiation in squamous epithelia [36] but takes on stemness-promoting activity upon malignant transformation [37]. The expression of NOTCH1 and $\beta$ catenin has been reported to be increased in $\mathrm{CD} 44^{+}$HNSCC cells [38]. CD133 (also known as AC133 or prominin-1) is a cell-surface glycoprotein comprising five transmembrane domains and two large glycosylated extracellular loops and has commonly been associated with subpopulations of cells 


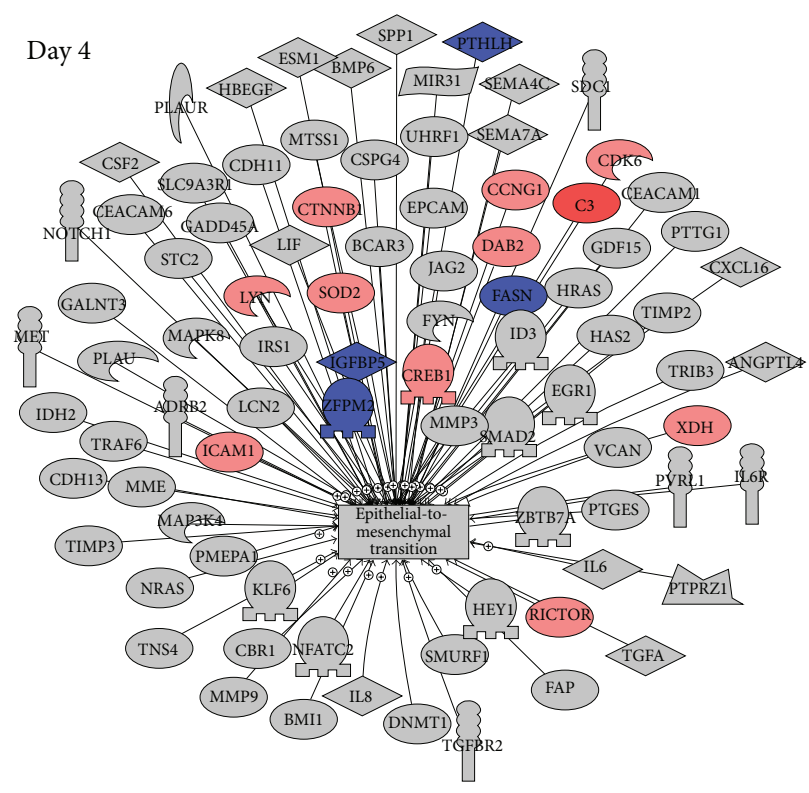

(a)

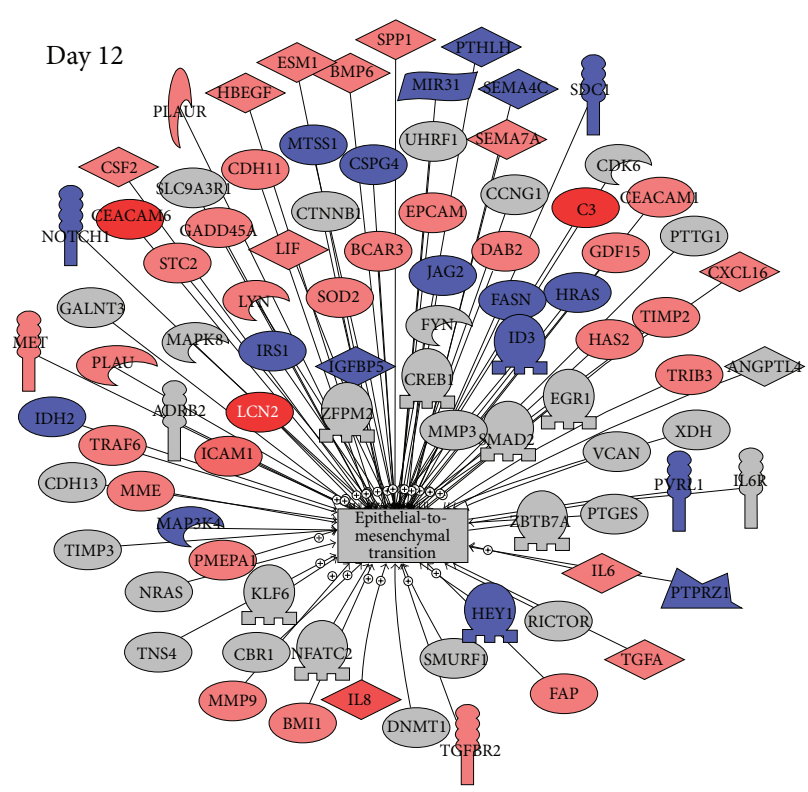

(c)

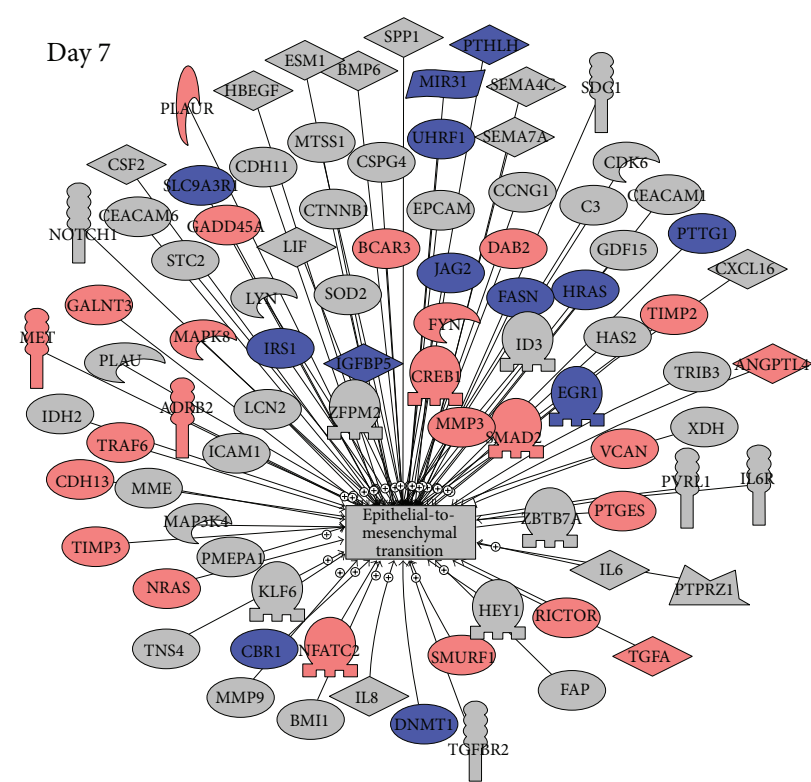

(b)

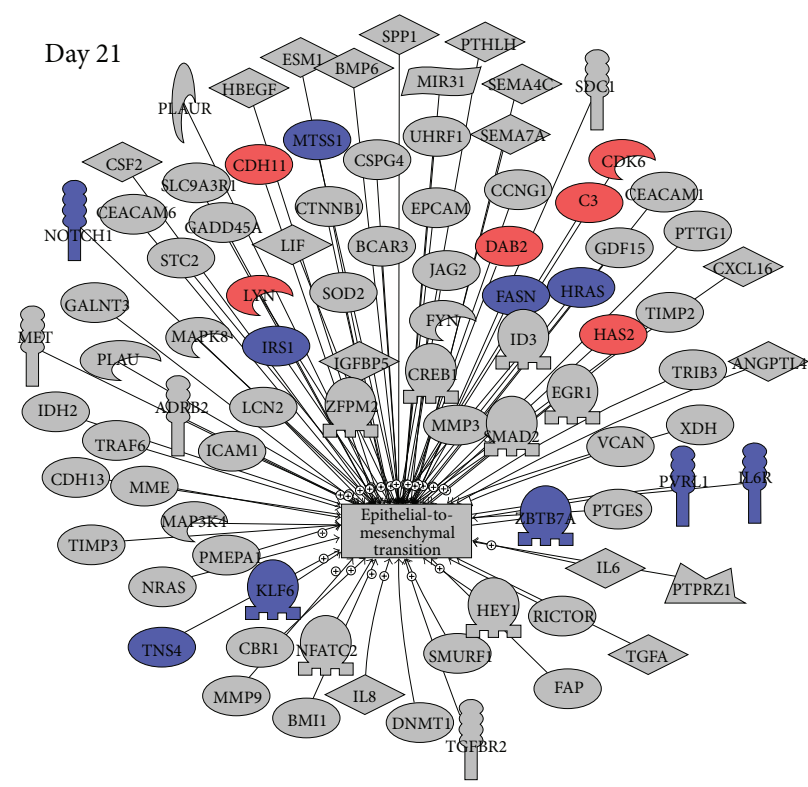

(d)

FIGURE 4: Changes in genes associated with epithelial-to-mesenchymal transition after radiation. Using Pathway Studio, genes known to be associated with epithelial-to-mesenchymal transition and differentially expressed at a minimum of one time point after radiation with a $p$ value $\leq 0.01$ and fold change (negative or positive) of 1.5 or greater were identified. Red represents genes upregulated after irradiation whilst blue represents downregulated genes.

with highly tumorigenic capacity in several cancers including HNSCC [39]. BMI1 is a member of the Polycomb family of transcription repressors. Emerging studies show that BMI1 has an important function as a biomarker of CSCs and is associated with self-renewal characteristics, tumor initiation, progression, invasion, metastasis, tumor recurrence, and resistance to chemotherapy and radiotherapy [40]. NANOG, POU5F1 (Oct-3/Oct-4), and SOX2 are transcription factors that are important in the maintenance of pluripotency and self-renewal in embryonic stem cells [41]. All these genes have been implicated in cancer stem cell traits in HNSCC [42].

There was a clear sequence of events that occurred in the signaling pathways for each of the stem cell-associated genes in that the number of significantly altered components increased from day 4 after irradiation to day 7 , peaked at day 12 , and returned to near control levels by day 21 . This sequence could be interpreted to represent the initial killing of radiation-sensitive cells, the recovery dominated 
by changes in the surviving radiation-resistant stem cell population, and then the return to normal activity driven by differentiation of stem cells into the original tumor phenotype. However, this is speculation as the changes in subpopulations of cells within the tumor cannot be verified by the current experimental design. The only principal gene that was upregulated at days 7 and 12 was MET (1.76- and 1.86fold, resp.), whilst the $A L D H 1$ family member $A L D H 1 A 3$ was upregulated at day $7(+2.18)$, day $12(+4.18)$, and day $21(+2.75)$. Both these genes also showed increased protein expression as evidenced by immunohistochemistry (Figure 2). BMI1 was modestly upregulated at day $12(+1.53)$ whilst NOTCH1 was downregulated at day $12(-2.11)$. The likely sequence of events following a single dose of $15 \mathrm{~Gy}$ would be initial killing of radiosensitive cells followed by a prolonged cell cycle delay with further rounds of cell death as cells attempt mitosis in the presence of unrepaired DNA damage. Accelerated repopulation during radiation has been described for many years [43] but the underlying stimulus for repopulation has not been well studied at the mechanistic level $[44,45]$. This is the first study to specifically look at stem cell signaling during the process of repopulation in an in vivo tumor model.

Each stem cell-associated gene was characterized by its own network of ligands, transcription factors, positive or negative regulators, or receptors which were significantly altered on different days after radiation treatment. However, there were genes that were common to many of the stem cell-associated genes including CTNNB1, MMP9, IL8, IL6, SMAD2, ICAM1, SPP1, TGFA, LIF, TGFBR2, and MAPK3. The key time period is likely to be the events and signaling that takes place between day 7 and day 12 . At this time, the tumor (and stromal) cells will be recovering from the single large radiation dose and the pathways that are activating the repopulation response will become dominant. This time period witnessed the most activity in terms of significantly altered genes linked with stem cell-associated genes as well as with the epithelial-to-mesenchymal transition. Interestingly, the NOTCH pathway did not seem to be activated following radiation; the gene $\mathrm{NOTCH} 1$ was downregulated at day 12 , one of its canonical transmembrane ligands JAG2 was downregulated at days 7 and 12, and its target gene $H E Y 1$ was also downregulated at day 12. The NOTCH pathway is one of the most intensively studied candidate genes involved in CSCs, and NOTCH signaling has been reported to promote the selfrenewal of CSC in several malignancies and to participate in tumor-stroma and tumor-endothelium interactions in CSC niches in primary and metastatic tumors [46]. Another gene which has been reported to be important in CSCs in head and neck cancer [40], BMI1, also did not show any significant changes during the repopulation response.

One of the most intriguing findings was centered on $A L D H 1$. This gene has been consistently associated with CSCs in head and neck cancer $[47,48]$. In our study ALDH1A3 was significantly upregulated at days 7,12 , and 21 as were the protein levels by immunohistochemistry (Figure 2), yet there were very few other family members that showed significant changes (Supplemental Table 4). ALDH is an intracellular enzyme that is normally active in the liver. Its primary functions are retinol conversion to retinoic acid and the oxidation of toxic aldehyde metabolites, such as those formed during the alcohol metabolism and certain chemotherapeutic drugs. It would seem to be a very significant biomarker for the identification of stem cells but offers little opportunities to design drugs to target its associated pathways. However, adoptive therapy with ALDH1A1-specific CD8(+) T cells is a promising approach to target CSCs based on this biomarker [49].

The MET/CD44 axis seems to be an important component of the CSC response in this HNSCC tumor model. Like ALDH1, MET gene expression and protein expression (Figure 2) were significantly altered at the key time points after irradiation. However, CD44 gene expression was not changed, and CD44 protein initially decreased following radiation before showing a recovery at day 12 . c-MET signaling plays a critical role in tumor progression, invasion, and metastasis, and we have recently shown it to be associated with poor prognosis in locally advanced HNSCC patients treated with chemoradiation [26]. Sun and Wang showed that c-MET(+) HNSCC cells increased expression of selfrenewal pathways, were spared by cisplatin treatment, and were responsible for mediating metastasis [35]. When CD44 expression was taken into account in addition to c-MET, the dual biomarker expressing population showed enhanced tumorigenicity [35]. A recent study showed that hepatocyte growth factor (the only known ligand for c-MET) stimulation increased the self-renewal and expression of stemness markers, such as Oct4, Sox2, Nanog, and CD44, in HNSCC stem-like cells [50]. In addition, knockdown of c-MET attenuated CSC traits, augmented cisplatin chemosensitivity, and inhibited xenotransplantation efficiency. Inhibiting c-MET using a kinase inhibitor, SU11274, inhibited sphere formation and suppressed the transcriptional levels of Oct 4 and Sox 2 of HNSCC stem-like cells [50]. It is difficult to decipher which are the key genes involved in the MET/CD44 axis, but IL- 8 has been shown to act as an autocrine growth factor in HNSCC, melanoma, and lung carcinoma and addition of recombinant IL-8 can promote directly the proliferation of HNSCC cell lines [51].

EMT is also linked with the acquisition of stem celllike characteristics. The concept of EMT inducing a CSC phenotype provides a possible mechanistic basis for metastasis, chemoresistance, tumor dormancy, and delayed recurrence. It is difficult to recognize the morphological cellular changes associated with EMT after radiation due to the cellular changes induced by the treatment which include enlarged nuclei with degenerative atypia, smudged chromatin, pyknotic nuclei, and micro-/macrovesicular vacuolation of cytoplasm [17]. However, the gene expression analysis highlighted the importance of EMT-associated genes in the repopulation response suggesting that this cellular process is a key component of the tumor response to recovery from cytotoxic damage.

In summary, stem cell-associated gene signaling is a key component of the response of HNSCC to DNA damage and may be driver of the repopulation response of the tumor. The limitation of this study is that experiments were performed on one tumor model and further work will be required using different HNSCC tumor models to validate these findings. In 
addition, the model used a single dose of irradiation; further work will be required using fractionation schedules with different total doses to confirm these findings in a clinically realistic schedule. With these limitations, this study indicates that the $M E T / C D 44$ axis would seem to be an important contributor to the recovery/regrowth response.

\section{Conclusion}

Although CSCs have been consistently identified in HNSCC and shown to be capable of enhancing tumorigenicity and resistance to chemotherapy, there has been no study that attempts to decipher their importance in the repopulation/regrowth response of HNSCC to radiation treatment. In this study, we have demonstrated that several pathways associated with known stem cell-associated genes show a similar response after irradiation that is consistent with the transition from damage recovery to regrowth of a HNSCC xenograft in vivo. The study highlights the potential importance of the $M E T / C D 44$ axis as well as the importance of epithelial-tomesenchymal regulators in the recovery process after DNA damage caused by radiation.

\section{Conflict of Interests}

The authors declare that there is no conflict of interests regarding the publication of this paper.

\section{References}

[1] J.-P. Pignon, A. L. Maître, E. Maillard, and J. Bourhis, "Metaanalysis of chemotherapy in head and neck cancer (MACH$\mathrm{NC}$ ): an update on 93 randomised trials and 17,346 patients," Radiotherapy and Oncology, vol. 92, no. 1, pp. 4-14, 2009.

[2] M. Bryne, M. Boysen, C. G. Alfsen et al., "The invasive front of carcinomas. The most important area for tumour prognosis?" Anticancer Research, vol. 18, no. 6, pp. 4757-4764, 1998.

[3] S. C. Tremmel, K. Götte, S. Popp et al., "Intratumoral genomic heterogeneity in advanced head and neck cancer detected by comparative genomic hybridization," Cancer Genetics and Cytogenetics, vol. 144, no. 2, pp. 165-174, 2003.

[4] M. P. Tabor, R. H. Brakenhoff, V. M. M. Van Houten et al., "Persistence of genetically altered fields in head and neck cancer patients: biological and clinical implications," Clinical Cancer Research, vol. 7, no. 6, pp. 1523-1532, 2001.

[5] T. Reya, S. J. Morrison, M. F. Clarke, and I. L. Weissman, "Stem cells, cancer, and cancer stem cells," Nature, vol. 414, no. 6859, pp. 105-111, 2001.

[6] Y.-C. Chen, Y.-W. Chen, H.-S. Hsu et al., "Aldehyde dehydrogenase 1 is a putative marker for cancer stem cells in head and neck squamous cancer," Biochemical and Biophysical Research Communications, vol. 385, no. 3, pp. 307-313, 2009.

[7] Z. G. Chen, "The cancer stem cell concept in progression of head and neck cancer," Journal of Oncology, vol. 2009, Article ID 894064, 8 pages, 2009.

[8] M. R. Loebinger, A. Giangreco, K. R. Groot et al., "Squamous cell cancers contain a side population of stem-like cells that are made chemosensitive by ABC transporter blockade," British Journal of Cancer, vol. 98, no. 2, pp. 380-387, 2008.
[9] R. Pries, N. Wittkopf, T. Trenkle, S. M. Nitsch, and B. Wollenberg, "Potential stem cell marker CD44 is constitutively expressed in permanent cell lines of head and neck cancer," In Vivo, vol. 22, no. 1, pp. 89-92, 2008.

[10] M. E. Prince, R. Sivanandan, A. Kaczorowski et al., "Identification of a subpopulation of cells with cancer stem cell properties in head and neck squamous cell carcinoma," Proceedings of the National Academy of Sciences of the United States of America, vol. 104, no. 3, pp. 973-978, 2007.

[11] P. Zhang, Y. Zhang, L. Mao, Z. Zhang, and W. Chen, "Side population in oral squamous cell carcinoma possesses tumor stem cell phenotypes," Cancer Letters, vol. 277, no. 2, pp. 227234, 2009.

[12] W. A. Woodward, M. S. Chen, F. Behbod, M. P. Alfaro, T. A. Buchholz, and J. M. Rosen, "WNT/beta-catenin mediates radiation resistance of mouse mammary progenitor cells," Proceedings of the National Academy of Sciences of the United States of America, vol. 104, no. 2, pp. 618-623, 2007.

[13] T. M. Phillips, W. H. McBride, and F. Pajonk, "The response of $\mathrm{CD} 24^{-/ \text {low }} / \mathrm{CD} 44^{+}$breast cancer-initiating cells to radiation," Journal of the National Cancer Institute, vol. 98, no. 24, pp. 1777$1785,2006$.

[14] D. Hambardzumyan, O. J. Becher, M. K. Rosenblum, P. P. Pandolfi, K. Manova-Todorova, and E. C. Holland, "PI3K pathway regulates survival of cancer stem cells residing in the perivascular niche following radiation in medulloblastoma in vivo," Genes and Development, vol. 22, no. 4, pp. 436-448, 2008.

[15] S. Bao, Q. Wu, R. E. McLendon et al., "Glioma stem cells promote radioresistance by preferential activation of the DNA damage response," Nature, vol. 444, no. 7120, pp. 756-760, 2006.

[16] N. Facompre, H. Nakagawa, M. Herlyn, and D. Basu, "Stemlike cells and therapy resistance in squamous cell carcinomas," Advances in Pharmacology, vol. 65, pp. 235-265, 2012.

[17] J. Huang, J. L. Chunta, M. Amin et al., "Detailed characterization of the early response of head-neck cancer xenografts to irradiation using ${ }^{18}$ F-FDG-PET imaging," International Journal of Radiation Oncology, Biology, Physics, vol. 84, no. 2, pp. 485491, 2012.

[18] A. Subramanian, P. Tamayo, V. K. Mootha et al., "Gene set enrichment analysis: A knowledge-based approach for interpreting genome-wide expression profiles," Proceedings of the National Academy of Sciences of the United States of America, vol. 102, no. 43, pp. 15545-15550, 2005.

[19] A. Nikitin, S. Egorov, N. Daraselia, and I. Mazo, "Pathway studio-the analysis and navigation of molecular networks," Bioinformatics, vol. 19, no. 16, pp. 2155-2157, 2003.

[20] E. Kotelnikova, N. Ivanikova, A. Kalinin, A. Yuryev, and N. Daraselia, "Atlas of signaling for interpretation of microarray experiments," PLoS ONE, vol. 5, no. 2, Article ID e9256, 2010.

[21] S. Novichkova, S. Egorov, and N. Daraselia, "MedScan, a natural language processing engine for MEDLINE abstracts," Bioinformatics, vol. 19, no. 13, pp. 1699-1706, 2003.

[22] A. Yuryev, Z. Mulyukov, E. Kotelnikova et al., "Automatic pathway building in biological association networks," $B M C$ Bioinformatics, vol. 7, article 171, 2006.

[23] F. Bhaijee, D. J. Pepper, K. T. Pitman, and D. Bell, "Cancer stem cells in head and neck squamous cell carcinoma: a review of current knowledge and future applications," Head \& Neck, vol. 34, no. 6, pp. 894-899, 2012.

[24] J. Chen, J. Zhou, J. Lu, H. Xiong, X. Shi, and L. Gong, "Significance of CD44 expression in head and neck cancer: a 
systemic review and meta-analysis," BMC Cancer, vol. 14, article $15,2014$.

[25] G. F. Huber, A. Albinger-Hegyi, A. Soltermann et al., "Expression patterns of Bmi-1 and p16 significantly correlate with overall, disease-specific, and recurrence-free survival in oropharyngeal squamous cell carcinoma," Cancer, vol. 117, no. 20, pp. 4659-4670, 2011.

[26] A. M. Baschnagel, L. Williams, A. Hanna et al., "c-met expression is a marker of poor prognosis in patients with locally advanced head and neck squamous cell carcinoma treated with chemoradiation," International Journal of Radiation Oncology Biology Physics, vol. 88, no. 3, pp. 701-707, 2014.

[27] D. Li, P. Dong, C. Wu, P. Cao, and L. Zhou, "Notch1 overexpression associates with poor prognosis in human laryngeal squamous cell carcinoma," Annals of Otology, Rhinology \& Laryngology, vol. 123, no. 10, pp. 705-710, 2014.

[28] C. Zhou and B. Sun, "The prognostic role of the cancer stem cell marker aldehyde dehydrogenase 1 in head and neck squamous cell carcinomas: a meta-analysis," Oral Oncology, vol. 50, no. 12, pp. 1144-1148, 2014.

[29] A. Schröck, M. Bode, F. J. M. Göke et al., "Expression and role of the embryonic protein SOX2 in head and neck squamous cell carcinoma," Carcinogenesis, vol. 35, no. 7, Article ID bgu094, pp. 1636-1642, 2014.

[30] S. Trapasso and E. Allegra, "Role of CD44 as a marker of cancer stem cells in head and neck cancer," Biologics: Targets and Therapy, vol. 6, pp. 379-383, 2012.

[31] A. Faber, C. Barth, K. Hörmann et al., "CD44 as a stem cell marker in head and neck squamous cell carcinoma," Oncology Reports, vol. 26, no. 2, pp. 321-326, 2011.

[32] X. Qian, S. Wagner, C. Ma et al., "Prognostic significance of ALDH1A1-positive cancer stem cells in patients with locally advanced, metastasized head and neck squamous cell carcinoma," Journal of Cancer Research and Clinical Oncology, vol. 140, no. 7, pp. 1151-1158, 2014.

[33] S. Krishnamurthy, Z. Dong, D. Vodopyanov et al., "Endothelial cell-initiated signaling promotes the survival and self-renewal of cancer stem cells," Cancer Research, vol. 70, no. 23, pp. 99699978, 2010.

[34] M.-J. De Herdt and R. J. Baatenburg de Jong, "HGF and c-MET as potential orchestrators of invasive growth in head and neck squamous cell carcinoma," Frontiers in Bioscience, vol. 13, no. 7, pp. 2516-2526, 2008.

[35] S. Sun and Z. Wang, "Head neck squamous cell carcinoma c$\mathrm{Met}^{+}$cells display cancer stem cell properties and are responsible for cisplatin-resistance and metastasis," International Journal of Cancer, vol. 129, no. 10, pp. 2337-2348, 2011.

[36] Y. S. Brooks, P. Ostano, S. H. Jo et al., "Multifactorial ER $\beta$ and NOTCH1 control of squamous differentiation and cancer," The Journal of Clinical Investigation, vol. 124, no. 5, pp. 2260-2276, 2014.

[37] S. H. Lee, H. S. Hong, Z. X. Liu et al., “TNFalpha enhances cancer stem cell-like phenotype via Notch-Hesl activation in oral squamous cell carcinoma cells," Biochemical and Biophysical Research Communications, vol. 424, no. 1, pp. 58-64, 2012.

[38] K. Chikamatsu, H. Ishii, G. Takahashi et al., "Resistance to apoptosis-inducing stimuli in CD44+ head and neck squamous cell carcinoma cells," Head and Neck, vol. 34, no. 3, pp. 336-343, 2012.

[39] Q. Zhang, S. Shi, Y. Yen, J. Brown, J. Q. Ta, and A. D. Le, "A subpopulation of $\mathrm{CD}_{133^{+}}$cancer stem-like cells characterized in human oral squamous cell carcinoma confer resistance to chemotherapy," Cancer Letters, vol. 289, no. 2, pp. 151-160, 2010.

[40] E. Allegra, S. Trapasso, D. Pisani, and L. Puzzo, "The role of BMI1 as a biomarker of cancer stem cells in head and neck cancer: a review," Oncology, vol. 86, no. 4, pp. 199-205, 2014.

[41] L. A. Boyer, I. L. Tong, M. F. Cole et al., "Core transcriptional regulatory circuitry in human embryonic stem cells," Cell, vol. 122, no. 6, pp. 947-956, 2005.

[42] Y. C. Lim, S.-Y. Oh, Y. Y. Cha, S.-H. Kim, X. Jin, and H. Kim, "Cancer stem cell traits in squamospheres derived from primary head and neck squamous cell carcinomas," Oral Oncology, vol. 47, no. 2, pp. 83-91, 2011.

[43] H. R. Withers, J. M. G. Taylor, and B. Maciejewski, "The hazard of accelerated tumor clonogen repopulation during radiotherapy," Acta Oncologica, vol. 27, no. 2, pp. 131-146, 1988.

[44] T. Nishioka, M. Yasuda, T. Takeshima et al., "Radiation-induced cancer cell repopulation: a possible mechanism implied by experiments using transplantable mouse-derived sarcoma cell line," Cell Structure and Function, vol. 36, no. 1, pp. 13-20, 2011.

[45] Q. Huang, F. Li, X. Liu et al., "Caspase 3-mediated stimulation of tumor cell repopulation during cancer radiotherapy," Nature Medicine, vol. 17, no. 7, pp. 860-866, 2011.

[46] I. Espinoza, R. Pochampally, F. Xing, K. Watabe, and L. Miele, "Notch signaling: targeting cancer stem cells and epithelial-tomesenchymal transition," OncoTargets and Therapy, vol. 6, pp. 1249-1259, 2013.

[47] P. S. Satpute, V. Hazarey, R. Ahmed, and L. Yadav, "Cancer stem cells in head and neck squamous cell carcinoma: a review," Asian Pacific Journal of Cancer Prevention, vol. 14, no. 10, pp. 55795587, 2013.

[48] M. R. Clay, M. Tabor, J. H. Owen et al., "Single-marker identification of head and neck squamous cell carcinoma cancer stem cells with aldehyde dehydrogenase," Head and Neck, vol. 32, no. 9, pp. 1195-1201, 2010.

[49] C. Visus, Y. Wang, A. Lozano-Leon et al., "Targeting ALDH ${ }^{\text {bright }}$ human carcinoma-initiating cells with ALDH1A1-specific $\mathrm{CD}^{+}$T cells," Clinical Cancer Research, vol. 17, no. 19, pp. 6174-6184, 2011.

[50] Y. C. Lim, H. J. Kang, and J. H. Moon, "C-Met pathway promotes self-renewal and tumorigenecity of head and neck squamous cell carcinoma stem-like cell," Oral Oncology, vol. 50, no. 7, pp. 633-639, 2014.

[51] S. H. Hong, F. G. Ondrey, I. M. Avis et al., "Cyclooxygenase regulates human oropharyngeal carcinomas via the proinflammatory cytokine IL-6: a general role for inflammation?" The FASEB Journal, vol. 14, no. 11, pp. 1499-1507, 2000. 

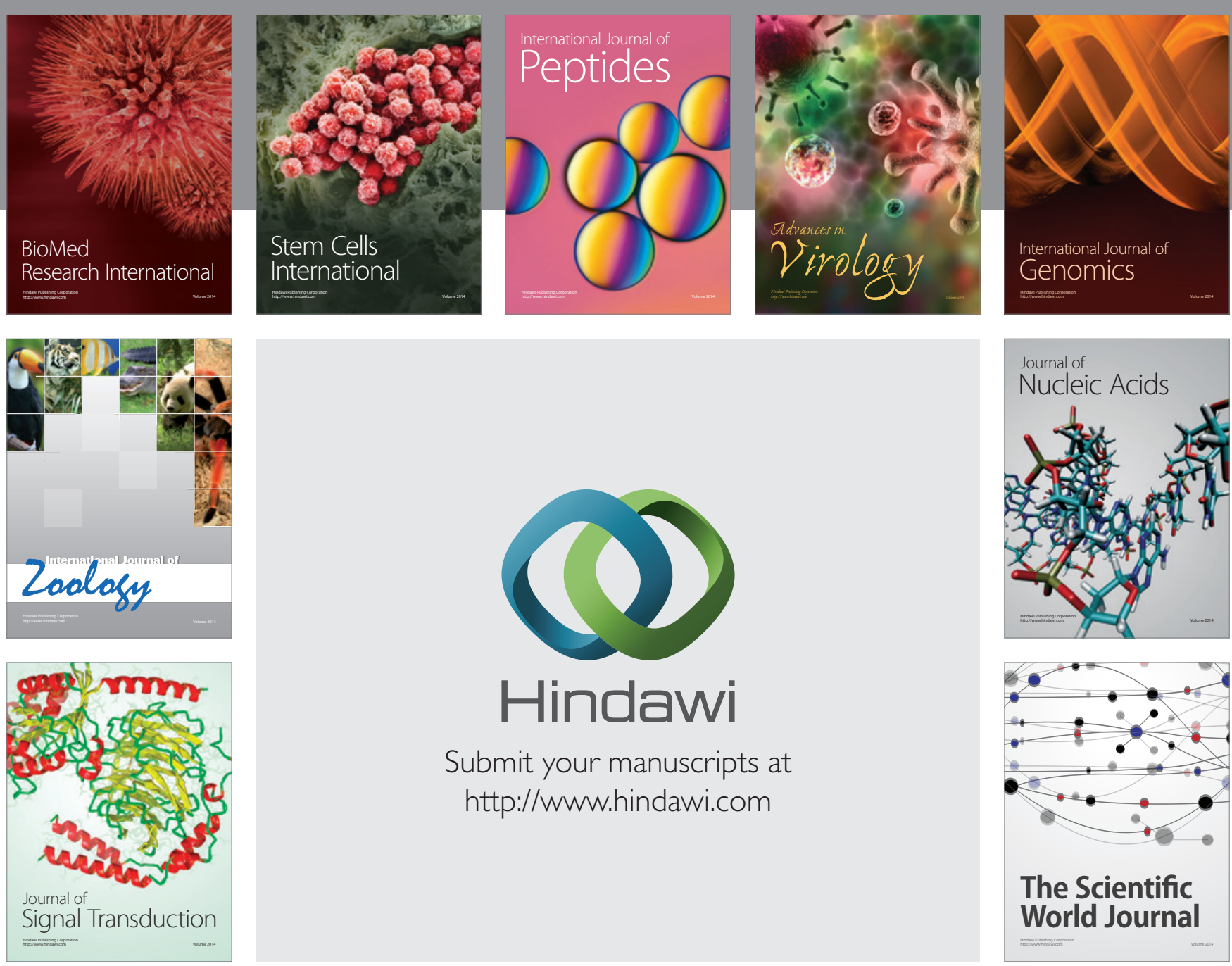

Submit your manuscripts at

http://www.hindawi.com
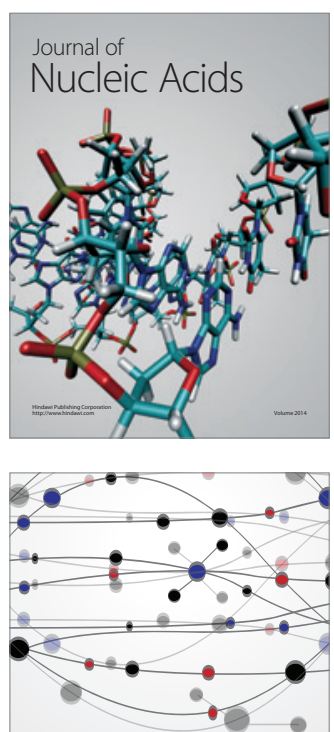

The Scientific World Journal
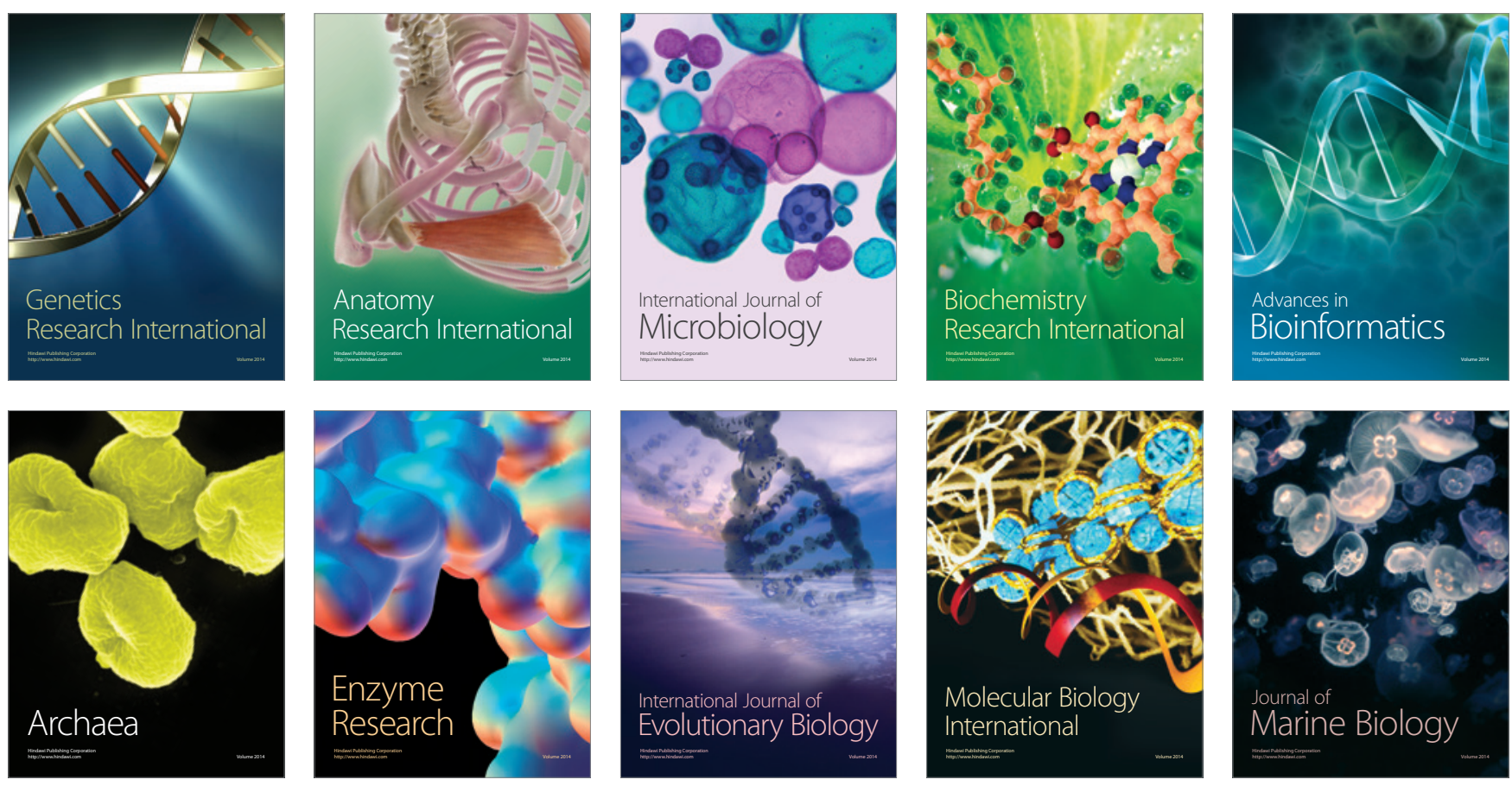